\title{
The Effect of Topic Familiarity on L2 Incidental Vocabulary Acquisition in Reading-An Experimental Study Based on Path Analysis
}

\author{
ZHANG Yu-jiao \\ Longdong University, Qingyang, China
}

\begin{abstract}
Among the current studies of the effect of topic familiarity on incidental vocabulary acquisition (IVA) in reading, reading has long been seen as a tool to achieve the experiment and reading performance has not been taken as a possible independent variable in the research. In view of this, this paper, with the help of Path Analysis in SPSS 17.0, explores the relationship between topic familiarity, reading performance, and L2 incidental vocabulary acquisition. It is found that topic familiarity has a significant influence on reading performance and reading performance has a significant effect on vocabulary learning. Specifically, topic familiarity has an indirect influence on IVA but reading performance has a direct significant effect on it.
\end{abstract}

Keywords: topic familiarity, reading performance, L2 vocabulary, incidental vocabulary acquisition (IVA)

\section{Introduction}

Vocabulary is the foundation of English learning, so how to acquire numbers of words in a limited time has been attracting the attention of learners and researchers. However, the researches into vocabulary now mainly focus on the incidental vocabulary acquisition (IVA) in reading (see Pulido, 2003; GAI, 2003, etc.) and all possible factors that may influence the vocabulary acquisition have been taken into consideration. Among the current researches, reading and reading tasks have been regarded as a tool to fulfill the experiments all along and the effect of reading performance has hardly been taken into consideration. Reading is a complicated cognitive process. Schemata theory (Carrel, 1987) believed that reading is the process in which readers interact their existing knowledge and the text information to comprehend a passage; topic familiarity, a form of content schema, reflects the familiarity of a reader's specific background or knowledge in a topic; Leeser (2007) found topic familiarity is one factor that influences reading comprehension. Pulido (2003) and ZHU Lian-hong (2007) showed it has a significant influence on reading comprehension. Nevertheless, it is still worthwhile to probe into the influence of topic familiarity on incidental vocabulary learning in reading for Chinese English learners in colleges remote from the capital cities, because an overwhelming majority of these learners have a poor mastery of English with low pass rate of CET 4 (College English Test Grade 4) and do not know how to learn vocabulary. Consequently, how to help these learners learn the words effectively becomes an urgent problem for English teachers and researchers. What is more, few researches into the effect of topic familiarity on L2 incidental vocabulary acquisition have been conducted

ZHANG Yu-jiao, lecturer, master, School of Foreign Languages and Literature, Longdong University. 
on these learners. As is reviewed above, reading has always been seen as a way to complete the experiment, except GAI Shu-hua (2003), which investigated the influence of reading purposes on IVA, so this research will also explore the role of reading performance in the vocabulary acquisition and the relationship among topic familiarity, reading performance, and IVA.

Therefore, this research, with the help of Path Analysis in SPSS 17.0, explores the relationship between topic familiarity, reading performance, and L2 IVA with the expectation to be helpful to the vocabulary teaching in lower-grade colleges far from the capital cities of the southwest China.

\section{Research Questions}

Based on the reviews above, this research will explore the following questions:

(1) Is topic familiarity helpful for Chinese English learners’ IVA in English reading?

(2) Does reading performance influence learner's L2 incidental vocabulary acquisition?

(3) What relationship does there remain among topic familiarity, reading performance, and L2 incidental vocabulary acquisition?

Participants in this research are 39 non-English major sophomores from a lower-grade college in the northwest of China.

The instruments used in this research are: (1) two expository texts with the similar text structure and numbers of words. Besides, one is significantly more familiar than the other to the participants (Sig. $=0.000$ ); (2) eight target words closely related to the subject to the texts, four in each text. In the experiment, to make sure all the words are completely new to all participants, these words are replaced with the corresponding meaningless words created on the rules of the word formations and pronunciations but with their inflectional and derivational forms (see also in Pulido, 2003; ZHANG, 2007; DAI, 2005); (3) questionnaire of topic familiarity with a rate scale of 1 to 5 to measure learner's familiarity of 10 topics, including the two in the research; and (4) translation test and selection test. Translation test requires learners to translate or define words without any clues from the text and it can be graded on the completely right answer with 1 point, partly right 0.5 point and wrong 0 , while selection test requires learners to choose the best alternative which can best explain the italicized words in a sentence from the text and it can be graded with the right choice with 1 point and the wrong one 0 point. Among the five choices of selection test, there is only one best answer, one answer is "I don't know" to stop learners from random guessing and another three interfering choices are either similar in meaning, in context, in spelling or pronunciation or related to the background knowledge of learners. Besides, target words also are used as choices in the test to avoid the interference of target words.

\section{Research Procedures}

To get the valid and reliable data in this research, firstly, questionnaires of topic familiarity are distributed to decide and choose the topics of reading materials; secondly, participants are required to read the two expositions and retell them in Chinese individually without any limitation of time. Besides, the performance of reading is graded on points of percentage; thirdly, participants are required to finish the test of translation and selection, translation tests are delivered to be done first and then are selection tests. More importantly, participants do not know they will be required to do the tests of vocabulary until they finish the reading and reading tasks. 


\section{Results and Discussion}

Valid and reliable data are finally from 39 participants and Path Analysis of SPSS 17.0 is used to analyze all the data.

Table 1 shows learners have acquired or learned some vocabularies in both translation and selection tests. Besides, learners could also be found to acquire more words in selection tests than in the translation tests ( $\mathrm{M}=$ $1.6154>0.2949$ ). This finding confirms and enriches the previous hypothesis and findings, in Knight (1994), Pulido (2003), and GAI Shu-hua (2003), for it indicates learners can acquire vocabulary no matter how proficient they are of English. Meanwhile, related information or context offered in the selection test assists learners to acquire more words than in the translation test with no context, which provides evidence to schema theory, that is, learners comprehend and construct the meaning of the reading materials with the assistance of the existing information.

Table 1

Descriptive Statistics of IVA in Both Translation Test and Selection Test

\begin{tabular}{llllllllll}
\hline & \multirow{2}{*}{$\mathrm{N}$} & \multicolumn{4}{c}{ Translation } & \multicolumn{3}{c}{ Selection } \\
\cline { 3 - 12 } & & Min & Max & Mean & Std. & Min & Max & Mean & Std. \\
\hline IVA & 78 & 0.00 & 2.50 & 0.2949 & 0.69989 & 0.00 & 4.00 & 1.6154 & 1.05966 \\
\hline
\end{tabular}

Topic familiarity could influence the acquisition of vocabulary (Pulido, 2003; DAI, 2005) but it might be possible that the performance of reading tasks could also influence the IVA in reading, so this research takes factors, i.e., topic familiarity and reading performance, into consideration, to further investigate the relationship among topic familiarity, reading performances, and vocabulary acquisition. With the help of Path Analysis, two significant pathways of the influence on vocabulary acquisition could be found from the Beta Coefficients in the path diagram in both translation test and selection test. Figure 1 shows one pathway is from topic familiarity to reading performance and then to vocabulary acquisition, in which topic familiarity influenced vocabulary acquisition via the intermediary variable, reading performance, so the influence of topic familiarity is indirect on vocabulary acquisition while that of reading performance is direct. The other pathway is from reading performance to vocabulary acquisition.

Beta $\left(=.324^{*}\right)$ demonstrates that topic familiarity influences reading performances directly and significantly, which approves learner's familiarity of a topic is a significant cause of the performance of reading tasks (Leeser, 2007). Given this, it is necessary for teachers to help learners be familiar with related topic or information in their reading teaching and to trigger the content schema to help learners read and comprehend in their reading. In the research, the importance of topic familiarity in reading comprehension is also stressed by learner's account that their lack of knowledge related to what they read results in their poor performance or even failure in the text understanding and word inference. Betas $\left(=.303^{*}, .263^{*}\right)$ indicate reading performance has a significant influence on vocabulary acquisition both in translation test and selection test, in other words, the better learner's performance of reading task is, the more vocabulary they can acquire. In any case, the finding in the path diagram, the pathways of significant influence from of topic familiarity on reading performance to reading performance on vocabulary acquisition, improve the researches (ZHU, 2007; DAI, 2005), more specifically, this research not only takes reading as a tool to accomplish the experimental purpose but it also researches the relationship among the intermediary variable reading performance, topic familiarity, and vocabulary acquisition. 


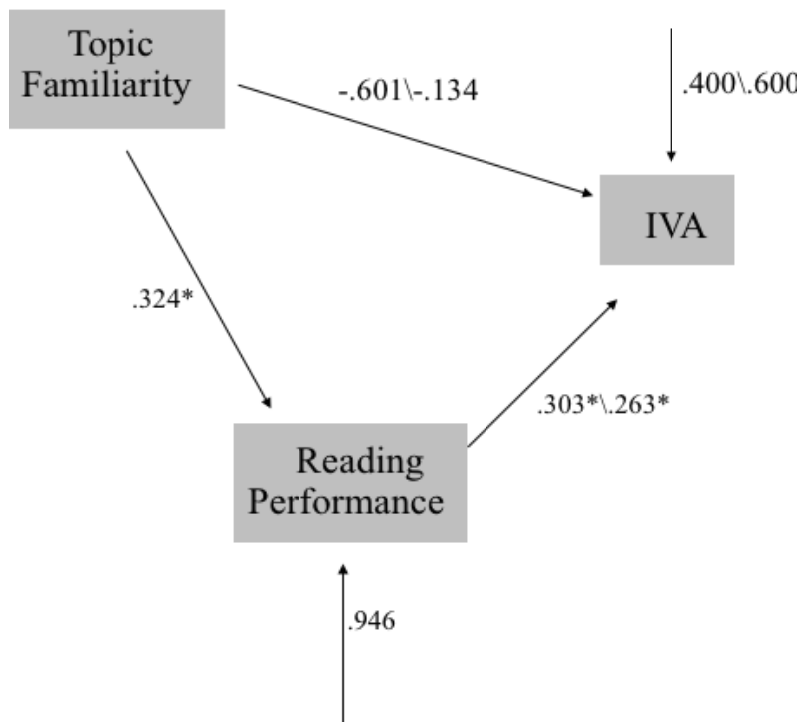

Figure 1. Path diagram. -.601, .303* refer to Betas in translation test; -.134, .263* are Betas in selection test; $.400, .600$ are residual coefficients respectively in translation and selection test.

It can be found in Figure 1 that the incidental acquisition of vocabulary can be affected directly by reading performance and indirectly by topic familiarity. Furthermore, the later random interview with learners found at least $18.5 \%$ participants in the research maintain that the poor performance of their reading tasks are largely due to their poor knowledge of the related background knowledge to the topic they read. In view of these, to assist learners to learn or acquire more vocabulary, it is the priority to help learners improve their reading proficiency; to improve their reading proficiency, they have to master the related knowledge and information to the topic of a text and activate and construct the corresponding schema, so teachers could consult the activities of introduction, discussion, questioning and prediction and use multimedia to help learners activate their existing schema before reading teaching. The finding that learners acquire more words in selection test than they do in translation test also proves the importance of background knowledge or the familiarity of a topic. However, the residual coefficients (.400 in translation test and .600 in selection test) in Figure 1 indicate IVA might also be affected by some other factors in addition to these two. What is more, though the participants in the research have at least 7-year-long experience of English learning as a foreign language, their English proficiency tends to be comparatively lower, compared with learners in those capital cities and vocabulary problem is one of the key problems among the obstacles in their English learning. Therefore, to improve these learners' vocabulary learning, the first thing is to improve their reading proficiency and to improve their reading proficiency means to help them with more related information and activate their existing knowledge.

\section{Conclusion}

Thanks to the previous theoretical and practical researches into L2 incidental vocabulary acquisition, this research can be inspired to investigate the relationship among topic familiarity, reading performance, and English incidental vocabulary acquisition through Path Analysis. It is found that topic familiarity can affect IVA via the intermediary variable of reading performance, that is, the influence of topic familiarity is indirect while that of reading performance is direct on English incidental vocabulary acquisition, so teachers are advised to activate learner's existing knowledge or schema by all means of possible activities, such as introduction, explanation, discussion, and questioning of the related information to the reading texts before reading to help 
them first with their reading performance and then further serve their vocabulary learning in reading process. Though the research is aimed highly, it is far from perfect, for example, the number of participants is too small so the finding might be further tested to see whether it is universal and practical to more learners.

\section{References}

Carrel, P. L. (1987). Content and formal schemata in EFL reading. TESOL Quarterly, 21, 461-481.

DAI, J. (2005). The influence of topic familiarity on vocabulary acquisition of second language in reading (Master's thesis). Guangdong University of Foreign Studies, Guangzhou, China.

GAI, S. H. (2003). An research into incidental vocabulary acquisition of Chinese English majors (Master's thesis). The PLA University of Foreign Languages, Luoyang, China.

Knight, S. (1994). Dictionary, the tool of last resort effects on comprehension and vocabulary acquisition for students of different verbal abilities. Modern Language Journal, 78, 285-298.

Leeser, M. J. (2007). Learner-based factors in L2 reading comprehension and processing grammatical form: Topic familiarity and working memory. Language Learning, 57(2), 229-270.

Pulido, D. (2003). Modeling the role of second language proficiency and topic familiarity in second language incidental vocabulary acquisition through reading. Language Learning, 53(2), 233-284.

ZHANG, J. M. (2007). The influence of topic familiarity on incidental vocabulary learning of Chinese English majors through reading (Master's thesis). Dalian University of Technology, Dalian, China.

ZHU, L. H. (2007). The influence of topic familiarity on incidental vocabulary acquisition of English. Journal of Zhejiang Sci-Tech University, 3, 350-353. 